\title{
Profilométrie par spectrométrie de masse d'ions secondaires de couches alternées cobalt-cuivre. Influence des conditions de pulvérisation sur la résolution en profondeur
}

\author{
François Jomard $\left({ }^{1}\right)$ et Marcel Perdereau $\left({ }^{2}\right)$ \\ $\left({ }^{1}\right)$ Crecamat-Centre régional de caractérisation des matériaux, 9 avenue A. Savary, \\ BP 400, 21011 Dijon Cedex, France \\ $\left(^{2}\right)$ UMR 5613 - CNRS-UB, 9 avenue A. Savary, BP 400, 21011 Dijon Cedex, France
}

(Reçu le 16 juillet 1997; accepté le 20 octobre 1997)

PACS.61.16.-d - Electron, ion, and scanning probe microscopy

Résumé. - Cet article présente les résultats obtenus par profilométrie SIMS sur des échantillons $\mathrm{Co} / \mathrm{Cu} / \mathrm{Co}$ et $\mathrm{Cu} / \mathrm{Co} / \mathrm{Cu}$. L'influence des conditions de bombardement par des ions $\mathrm{O}_{2}^{+}$ de $3 \mathrm{keV}$ (angle d'incidence et soufflage à l'oxygène) sur les résolutions en profondeur a été déterminée. La meilleure résolution en profondeur est obtenue sous incidence normale. Sous incidence tangentielle, la résolution en profondeur initialement très mauvaise, est notablement améliorée par le soufflage à l'oxygène. Le développement d'une rugosité sous l'effet de la pulvérisation est responsable de la dégradation de la qualité des profils. L'incorporation d'oxygène dans les matériaux métalliques, soit directement par bombardement d'ions $\mathrm{O}_{2}^{+}$sous incidence normale, soit par soufflage à l'oxygène, réduit considérablement le développement de la rugosité. Il est également présenté une différence entre le cuivre et le cobalt au point de vue rugosité après pulvérisation ionique.

\begin{abstract}
Depth resolution in SIMS profiling of multilayers $\mathrm{Co} / \mathrm{Cu} / \mathrm{Co}$ and $\mathrm{Cu} / \mathrm{Co} / \mathrm{Cu}$ samples has been estimated as a function of bombardment parameters with $3 \mathrm{keV} \mathrm{O}{ }_{2}^{+}$ions. The best depth resolution is obtained at normal incidence. At glancing incidence it is necessary to flood with oxygen to improve the resolution. Topography development due to sputtering, accountable for worsening of profiles, is reduced by oxygen implantation either directly by $\mathrm{O}_{2}^{+}$ at normal incidence or by flooding at glancing incidence.
\end{abstract}

\section{Introduction}

La profilométrie par SIMS de multicouches métalliques a donné matière à de nombreuses publications (par exemple [1-4]). Lors de l'utilisation des ions primaires $\mathrm{O}_{2}^{+}$, il a été souligné que l'obtention d'une bonne résolution en profondeur nécessitait l'adoption d'une faible énergie primaire associée à un soufflage à l'oxygène durant la pulvérisation. La majorité de ces expériences a été réalisée avec un appareillage ne permettant pas des variations indépendantes de l'énergie et de l'angle d'incidence des ions primaires.

Disposant d'une microsonde ionique (MIQ 256 Cameca - Riber), pour laquelle les paramètres de pulvérisation peuvent être fixés de façon indépendante, nous avons entrepris une 


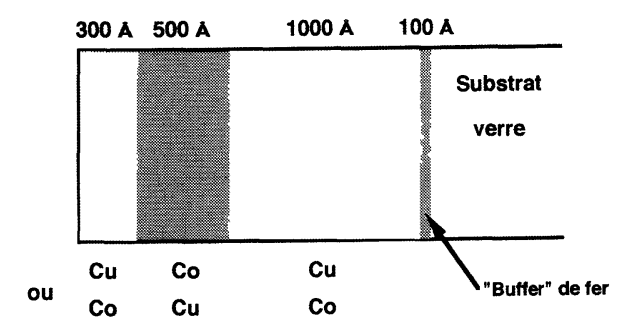

Fig. 1. - Structure des échantillons cobalt-cuivre.

[Cobalt-copper samples structure.]

étude systématique de l'influence de ceux ci sur les résolutions en profondeur obtenues sur des multicouches métalliques.

Cette étude a été réalisée dans le cadre du GDR G1108 du CNRS "Caractérisation des interfaces dans les multimatériaux". Les multicouches métalliques $\mathrm{Co} / \mathrm{Cu}$ ont été élaborées au Laboratoire de Physique des Solides de Bellevue. Les dépôts ont été effectués par pulvérisation cathodique RF sous des pressions contrôlées d'argon. Les structures de ces multicouches sont représentées figure 1. Le "buffer" de fer entre le substrat de verre et le dépôt favorise une texture bien définie. Les éléments $\mathrm{Co}$ et $\mathrm{Cu}$, quoique très voisins dans la classification périodique, possèdent des vitesses de pulvérisation différentes. Ce fut un des éléments du choix de ces matériaux.

Dans le cadre du GDR, ces échantillons ont été caractérisés avec des approches expérimentales différentes, soit destructives (SIMS et AES), soit non destructives (RBS - microsonde électronique). Nous ne présentons ici que l'aspect méthodologique de la profilométrie SIMS réalisée par CRECAMAT (centre régional de caractérisation des matériaux) à Dijon.

Pour toutes les exploitations, les épaisseurs nominales $(30 \mathrm{~nm}$ et $50 \mathrm{~nm}$ ) ont été admises. Ces interfaces n'ayant pas été caractérisées à l'échelle atomique (MET sur coupes polies) nous ne pouvons déduire de notre expérimentation la fonction de résolution en profondeur. Néanmoins toute amélioration dẹ la résolution en profondeur, mesurée sur un échantillon donné, correspond à une amélioration des conditions expérimentales.

\section{Expérimentation}

L'appareillage utilisé est une microsonde ionique MIQ 256 Cameca - Riber. L'analyse des ions secondaires est réalisée par un spectromètre de masse quadripolaire (gamme de masse 1-300). Les ions primaires réactifs $\mathrm{O}_{2}^{+}$ont été choisis de façon à augmenter globalement les intensités des signaux métalliques. Dans toute cette étude l'énergie a été fixée à $3 \mathrm{keV}$ de façon à obtenir un compromis acceptable entre une bonne qualité de faisceau et une énergie relativement faible conduisant à minimiser l'épaisseur perturbée par les ions. Dans l'appareillage utilisé et pour cette énergie constante de $3 \mathrm{keV}$, l'angle d'incidence par rapport à la normale de l'échantillon peut varier de $0^{\circ}$ à $70^{\circ}$. Le faisceau d'ions balaie sur l'échantillon une surface de l'ordre de $110 \times 110 \mu \mathrm{m}^{2}$, variable en fonction de l'angle d'incidence. Les intensités des ions secondaires $\left(\mathrm{O}^{+}, \mathrm{Co}^{+}, \mathrm{Cu}^{+}, \mathrm{CoCu}^{+}, \mathrm{Co}_{2}^{+}, \mathrm{Cu}_{2}^{+}\right)$sont mesurées, grâce à une fenêtre électronique de comptage, pendant le balayage d'une faible partie du centre du cratère de pulvérisation (5\%). Les conditions expérimentales sont reportées tableau I et un exemple de profil est présenté figure 2 . 


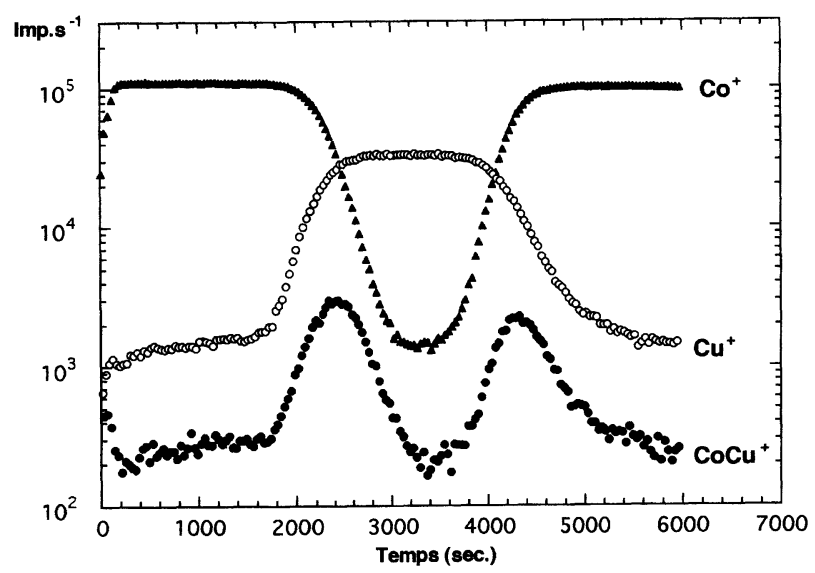

Fig. 2. - Un exemple de profil sur un échantillon $\mathrm{Co} / \mathrm{Cu} / \mathrm{Co}$.

[Depth profile obtained on a $\mathrm{Co} / \mathrm{Cu} / \mathrm{Co}$ sample.]

Tableau I. - Conditions expérimentales.

[Experimental conditions.]

\begin{tabular}{|l|l|}
\hline Ions primaires & $\mathrm{O}_{2}^{+}$ \\
\hline Energie primaire & $3 \mathrm{keV}$ \\
\hline Courant primaire & $5 \mathrm{nA}$ \\
\hline Surface balayée & $110 \times 110 \mu \mathrm{m}^{2}$ (incidence normale) \\
\hline Densité primaire & $40 \mu \mathrm{Acm}{ }^{-2}$ (incidence normale) \\
\hline Angle d'incidence & variable \\
\hline Fenêtre de comptage & $5 \%$ \\
\hline Temps de comptage & $250 \mathrm{~ms}$ \\
\hline Ions secondaires & ${ }^{16} \mathrm{O}^{+},{ }^{59} \mathrm{Co}^{+},{ }^{63} \mathrm{Cu}^{+},{ }^{118} \mathrm{Co}_{2}^{+},{ }^{126} \mathrm{Cu}_{2}^{+},{ }^{122} \mathrm{CoCu}^{+}$ \\
\hline
\end{tabular}

\section{Exploitation des résultats}

Les échantillons utilisés dans cette étude peuvent être considérés comme des métaux purs $(\mathrm{Co}, \mathrm{Cu})$ d'épaisseurs connues séparés par des interfaces que nous supposons abruptes. Les données expérimentales sont des variations de signaux en fonction du temps. De façon usuelle, les interfaces sont positionnées au temps $\left(t_{1 / 2}\right)$ pour lequel les signaux $\mathrm{Co}^{+}, \mathrm{Cu}^{+}$sont égaux à la moitié de leurs valeurs maximales. Ceci permet, compte tenu des épaisseurs admises, la détermination des vitesses de pulvérisation des matériaux purs.

Dans un premier temps nous avons "qualifié" les profils par une mesure de la résolution en profondeur $(\Delta z)_{16}^{84}$, épaisseur pour laquelle les signaux varient de $84 \%$ à $16 \%$ de leurs valeurs maximales. Dans l'étude présentée ici, cette procédure pose problème dans la mesure où :

1) les signaux ne varient pas de façon strictement concomitante, il en résulte une incertitude de quelques nm sur la position de l'interface ;

2) lors du passage de l'interface la vitesse de pulvérisation varie. Ici le rapport de la vitesse de pulvérisation du cuivre sur celle du cobalt peut atteindre 4. Pour la transformation de 


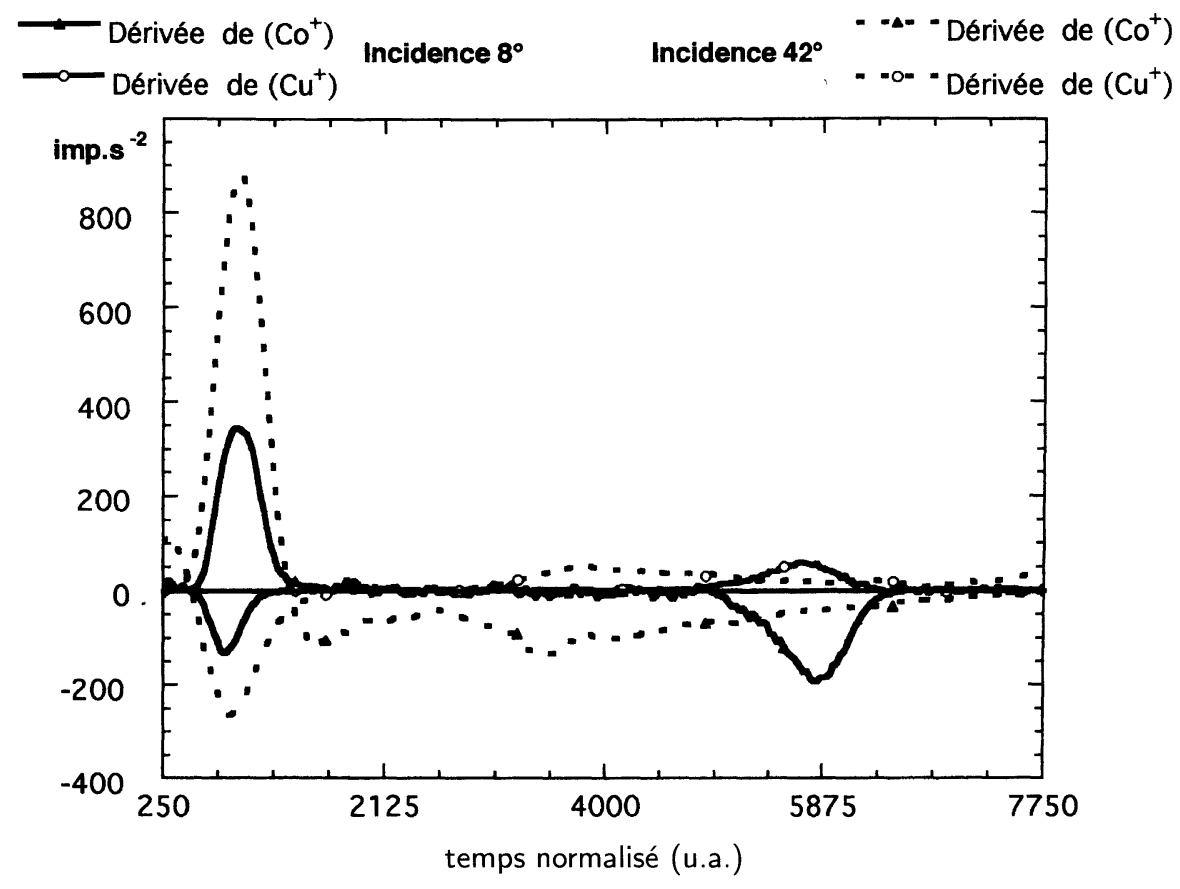

Fig. 3. - Dérivées des signaux $\mathrm{Cu}^{+}$et $\mathrm{Co}^{+}$pour deux profils obtenus sous des angles d'incidences différents. Noter sur le profil obtenu sous incidence tangentielle la disparition de la deuxième interface.

[Derivatives of the $\mathrm{Cu}^{+}$and $\mathrm{Co}^{+}$intensities versus time for two profiles obtained with two incidence angles. The second interface disappear at glancing incidence].

l'échelle des temps en échelle des profondeurs au voisinage de l'interface nous avons utilisé une vitesse de pulvérisation moyenne expérimentale, déterminée pour chaque profil. Ceci nous permet d'accéder à un $\left(\Delta z_{\text {moyen }}\right)_{16}^{84}$.

La résolution moyenne ainsi définie est toujours applicable mais elle est nettement insuffisante pour "qualifier" valablement et complètement un profil. Certains éléments doivent en effet être pris en compte notamment :

1) l'obtention de signaux constants dans le domaine d'existence des matériaux purs ;

2) une égalité des signaux entre différentes couches d'un même matériau (ici toujours deux avec les échantillons utilisés) ;

3) une grande variation des signaux $\mathrm{Cu}^{+}$et $\mathrm{Co}^{+}$lors de la traversée de l'interface (grande dynamique).

Une représentation des dérivées des signaux en fonction du temps permet un accès direct (et objectif) à l'existence de plateaux biens définis (Fig. 3). Les interfaces se matérialisent sur ces représentations par des variations des dérivées de part et d'autre de la valeur nulle. Les maxima des valeurs des dérivées varient comme la dynamique des signaux. À l'aide de ces courbes la résolution pourrait être définie comme la largeur à mi-hauteur des dérivées (FWHM). Quoiqu'il en soit les grandeurs ainsi définies $\left(\Delta z_{\text {moyen }}\right)_{16}^{84}$ et (FWHM) des dérivées évoluent dans le même sens avec les conditions expérimentales. Il faut remarquer que dans ces modes d'exploitation seule une faible dynamique de la variation des signaux est utilisée. Compte tenu du fait que les échantillons ne sont pas caractérisés à l'échelle atomique, la détermination des longueurs d'atténuation des signaux en coordonnées logarithmique ne sera pas rapportée ici, l'interprétation en étant "délicate". 


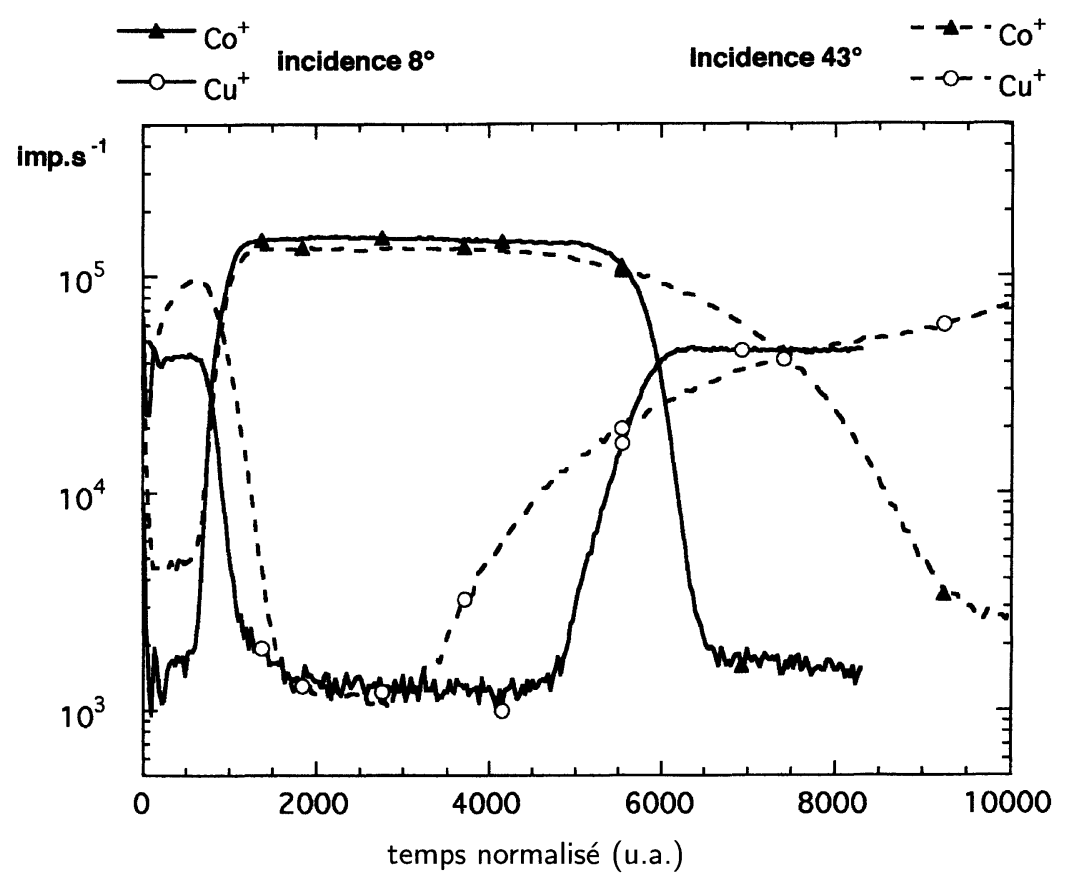

Fig. 4. - Influence de l'angle d'incidence des ions de pulvérisation sur deux profils obtenus sur un même échantillon $\mathrm{Cu} / \mathrm{Co} / \mathrm{Cu}$.

[Comparison of two profiles obtained with two incidence angles of the primary ions.]

\section{Résultats expérimentaux}

\subsection{Influence de l'angle d'incidence des ions primaires}

Cette étude a été réalisée sur des échantillons $\mathrm{Cu} / \mathrm{Co} / \mathrm{Cu}$. La figure 4 présente la superposition de deux profils réalisés sous deux incidences $\left(8^{\circ}\right.$ et $\left.43^{\circ}\right)$. Les échelles de temps ont été normalisées de façon à assurer la coïncidence des interfaces. On peut constater que la résolution s'améliore lorsque l'incidence se rapproche de la normale. La perte de résolution aux grands angles d'incidence est associée à la disparition des plateaux de signaux ainsi qu'à des valeurs différentes des maxima des signaux pour les couches de cuivre qui encadrent la couche de cobalt. Pour les incidences au voisinage de $50^{\circ}$, la deuxième interface $(\mathrm{Co} \rightarrow \mathrm{Cu}$ ) n'est pratiquement plus mise en évidence. Une analyse de la résolution en $\left(\Delta z_{\text {moyen }}\right)_{16}^{84}$, qui est à sa limite de validité pour les grands angles d'incidence, a été effectuée. Les variations de $\left(\Delta z_{\text {moyen }}\right)_{16}^{84}$ en fonction de l'angle sont reportées figure 5 .

Les vitesses de pulvérisation du cuivre et du cobalt varient en fonction de l'angle d'incidence des ions primaires. La figure 6 présente ces variations. Il s'agit d'expériences effectuées avec une intensité primaire constante. Lors de la modification de l'angle, la densité varie en $\cos (\theta)$. En admettant une variation du taux de pulvérisation $S(\theta)$ de la forme $S(\theta)=S(0) \cos (\theta)^{-n}$ (avec $n \sim 1$ ), les vitesses de pulvérisation devraient être constantes. Avec l'ion réactif $\mathrm{O}_{2}^{+}$cette hypothèse n'est pas vérifiée.

Les concentrations atomiques des métaux cobalt et cuivre sont très voisines $(8,45$ et $9,09 \times 10^{+22}$ atomes $\left.\mathrm{cm}^{-3}\right)$. Les vitesses de pulvérisation de ces deux métaux étant déterminées 


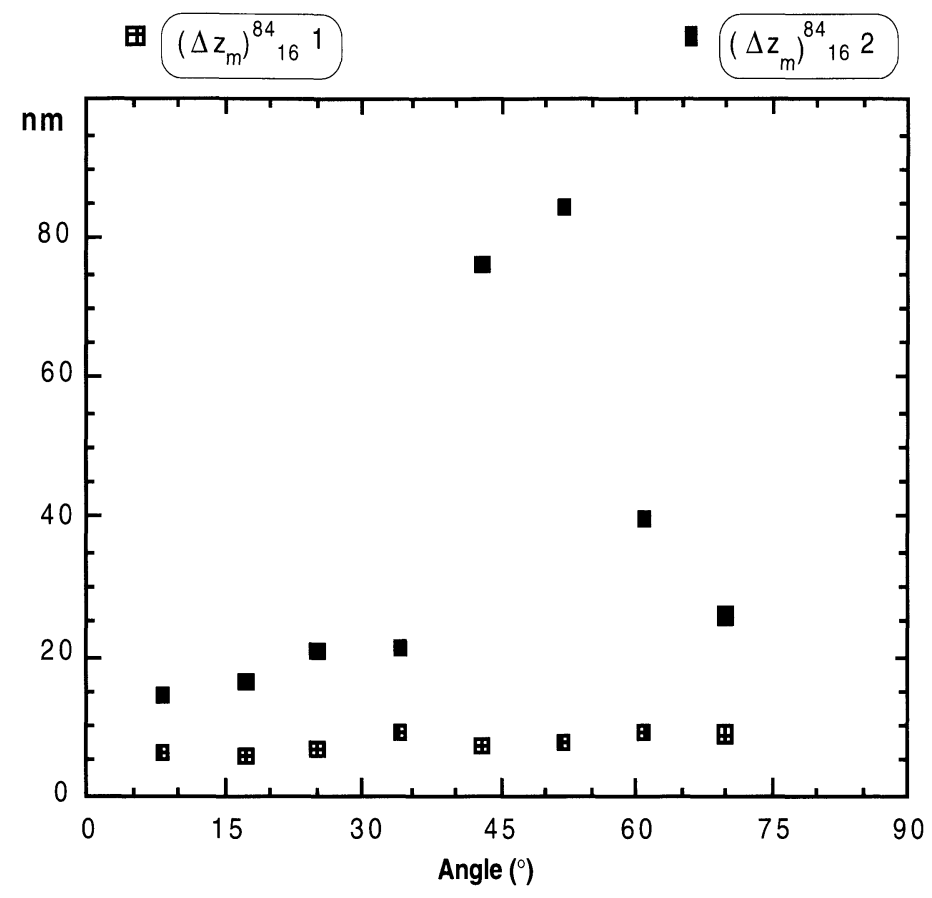

Fig. 5. - Résolutions moyennes $\left(\Delta z_{\text {moyen }}\right)_{16}^{84}$ pour les deux interfaces en fonction de l'angle d'incidence. Déterminations effectuées à $\pm 0,5 \mathrm{~nm}$.

[Mean depth resolution $\Delta z_{\text {mean }}$ for the two interfaces as a function of incidence angle. Experimental error $\pm 0.5 \mathrm{~nm}$.]

dans les mêmes conditions, le rapport des vitesses est assimilable au rapport des taux de pulvérisation. Le rapport des vitesses, donc des taux de pulvérisation varie en fonction de l'angle. Cette variation est présentée figure 7. Les rapports déterminés expérimentalement s'éloignent notablement des rapports théoriques des taux de pulvérisation calculés par simulation TRIM ou Profil Code.

Ces résultats concernant les rapports des vitesses de pulvérisation sont concordants avec d'autres déterminations obtenues dans les mêmes conditions d'angle d'incidence mais avec des appareillages différents [5].

Il est à noter que la meilleure résolution en profondeur est obtenue dans des conditions où les vitesses de pulvérisation sont les plus faibles.

\subsection{Influence du soufflage à l'oxygène}

Ces expériences ont été réalisées en introduisant au voisinage immédiat de l'échantillon par une fine tubulure $(\varnothing=1 \mathrm{~mm}$ ) de l'oxygène pur (air liquide-N45). L'exposition est repérée et ajustée grâce à une mesure de la pression dans la chambre d'analyse. La jauge étant placée très loin de l'échantillon l'exposition à l'oxygène est sous-estimée. Le vide limite de l'appareillage est égal à $2 \times 10^{-10}$ torr. La pression maximale, pour des raisons de compatibilité avec le système de pompage ionique, est fixée à $2 \times 10^{-6}$ torr. Plusieurs profils ont été réalisés avec et sans soufflage pour des incidences différentes $\left(8^{\circ}\right.$, incidence presque normale et $52^{\circ}$, incidence tangentielle). 


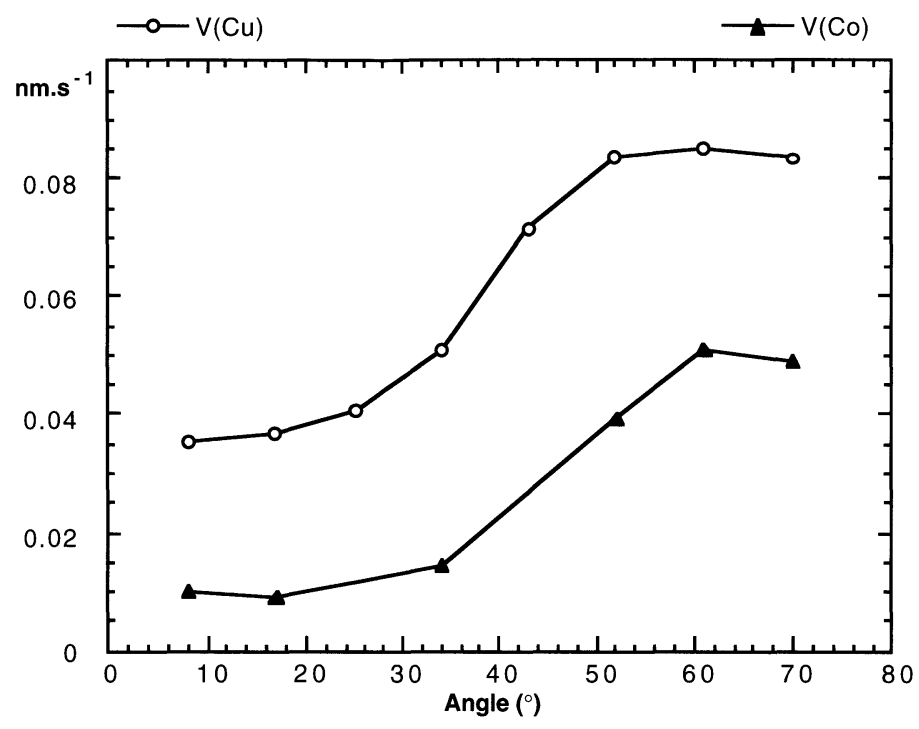

Fig. 6. - Vitesses de pulvérisation du cuivre et du cobalt en fonction de l'angle d'incidence.

[Sputtering rates of copper and cobalt as a function of incidence angle.]

Sous incidence tangentielle l'amélioration de la résolution en profondeur est très importante. Ceci est présenté figure 8 ou deux profils (incidence $52^{\circ}$ ), l'un sous UHV l'autre sous $P\left(\mathrm{O}_{2}\right)=$ $2 \times 10^{-6}$ torr, sont superposés. Les $\left(\Delta z_{\text {moyen }}\right)_{16}^{84}$ en fonction de la pression de soufflage sont reportés sur la figure 9 .

Le soufflage à l'oxygène augmente les intensités des ions secondaires $\mathrm{Co}^{+}$et $\mathrm{Cu}^{+}$(Fig. 10). L'augmentation est plus importante pour l'ion $\mathrm{Co}^{+}$; ceci est normal, l'élément cobalt étant plus électropositif que l'élément cuivre $(E(\mathrm{Cu})=1,9, E(\mathrm{Co})=1,8)$ et ayant une affinité plus grande pour l'oxygène $\left(\Delta H_{\mathrm{f}}^{0}\right.$ par mole d'O-Co $\left.\sim-230 \mathrm{~kJ} \mathrm{~mol}^{-1} / \mathrm{Cu} \sim-150 \mathrm{~kJ} \mathrm{~mol}^{-1}\right)$. Il faut remarquer que l'augmentation par soufflage de l'intensité de l'ion $\mathrm{O}^{+}$est plus importante dans la matrice cobalt que dans la matrice cuivre (Fig. 11). L'incorporation de l'oxygène due au soufflage entraîne une diminution des vitesses de pulvérisation avec une augmentation du rapport $V_{\mathrm{p}}(\mathrm{Cu}) / V_{\mathrm{p}}(\mathrm{Co})$ qui tend vers le rapport obtenu sous l'incidence normale et sous ultravide (Fig. 12).

Sous incidence normale le soufflage par l'oxygène n'améliore pas la résolution moyenne dans la limite des erreurs expérimentales. On relève uniquement une augmentation des ions secondaires $\mathrm{Co}^{+}$et $\mathrm{Cu}^{+}$, plus importante pour le $\mathrm{Co}^{+}$.

\subsection{Mesures des rugosités}

Les variations de résolution en profondeur pouvant être attribuées à des rugosités induites lors de la pulvérisation nous avons commencé une étude systématique de celles-ci.

Deux techniques ont été utilisées, l'examen au microscope électronique à balayage et la mesure des topographies par microscopie AFM. Ces deux techniques ont été appliqués à des fonds de cratères de pulvérisation effectués sous des conditions différentes, correspondant à des résolutions moyennes en profondeur mesurées et variables. Les épaisseurs pulvérisées sont 


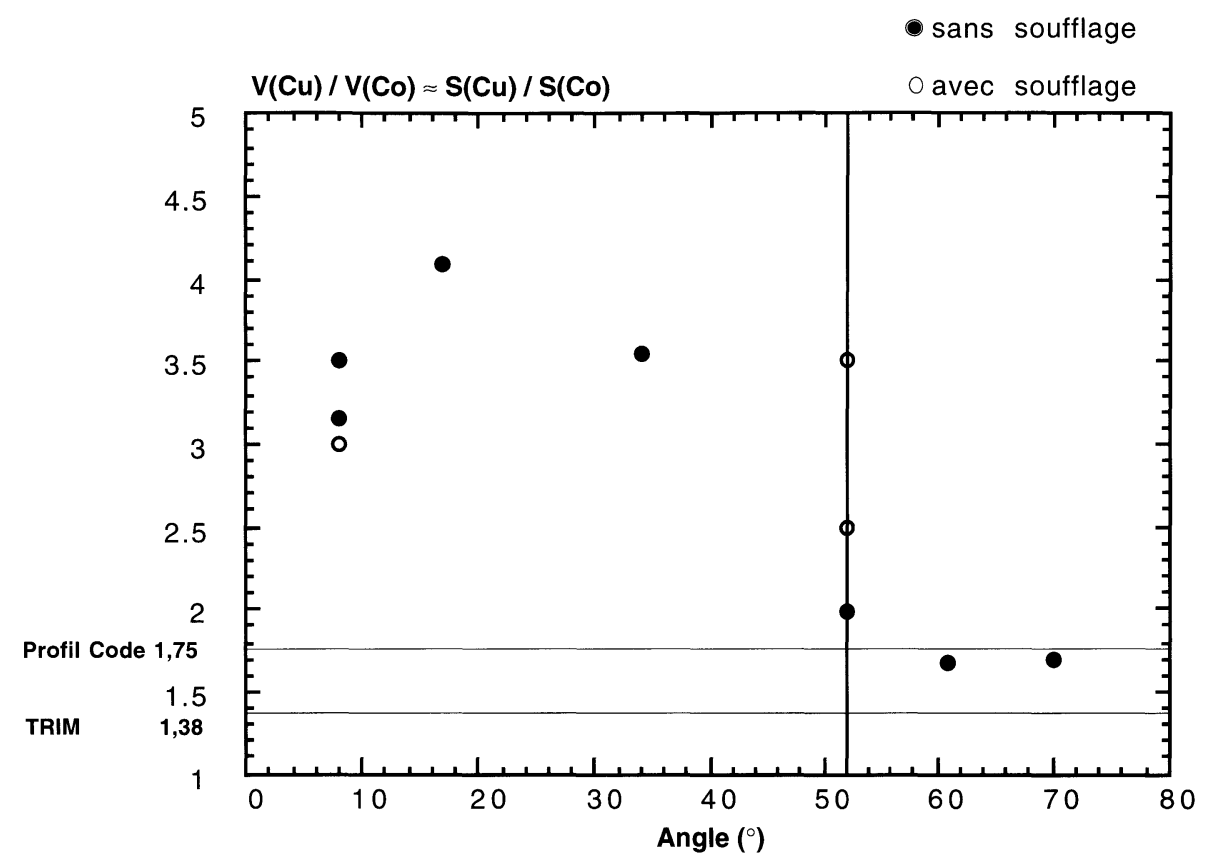

Fig. 7. - Évolution du rapport de la vitesse de pulvérisation du cuivre sur celle du cobalt en fonction de l'angle d'incidence. Les valeurs du rapport des taux de pulvérisation obtenus par simulation (TRIM et Profil Code) sont également reportées sur la figure.

[Variation of sputtering rates ratio of copper/cobalt as a function of incidence angle. The calculated ratio (TRIM and Profil Code) for the sputtering yield are reported on the figure.]

toutes de l'ordre de $100 \mathrm{~nm}$, ce qui positionne le fond de cratère au delà de la deuxième interface. Les doses d'ions reçues sont de l'ordre de $1 \times 10^{+18}$ ions $\mathrm{cm}^{-2}$. Du fait de la nature des échantillons le matériau est soit du cobalt soit du cuivre.

Les examens microscopiques ont été effectués sur un microscope électronique à balayage JEOL 6400F. La composition des fonds de cratères étant monoélémentaire, seules des images électroniques secondaires, donnant un contraste de topographie, sont envisageables. Pour les surfaces peu rugueuses, donc peu contrastées, la mise au point est délicate. Il en découle que ne peuvent être valablement visualisés que les fonds de cratères rugueux, obtenus sous incidence tangentielle et correspondant à des profils pour lesquels la résolution en profondeur est mauvaise. Les deux matériaux présentent des aspects très différents. Le cuivre (Fig. 13a) présente des cônes, dispersés uniformément, orientés dans la même direction avec un diamètre à la base de l'ordre de $100 \mathrm{~nm}$. Le cobalt (Fig. 13b) présente une stucture apparemment beaucoup moins rugueuse.

Les mesures de rugosité ont été effectuées à l'aide d'un microscope AFM. (Nanoscope II Digital Instruments) fonctionnant en mode contact. Cette technique permet outre l'obtention d'images, non présentées ici, des relevés de profils et une analyse statistique de la topographie. La figure 14 présente deux profils se rapportant aux images de MEB et montre que les deux techniques sont concordantes : le cuivre présente une rugosité plus importante que le cobalt et le profil confirme la présence de cônes avec des diamètres à la base de l'ordre de $100 \mathrm{~nm}$ et des hauteurs pouvant atteindre $30 \mathrm{~nm}$. 

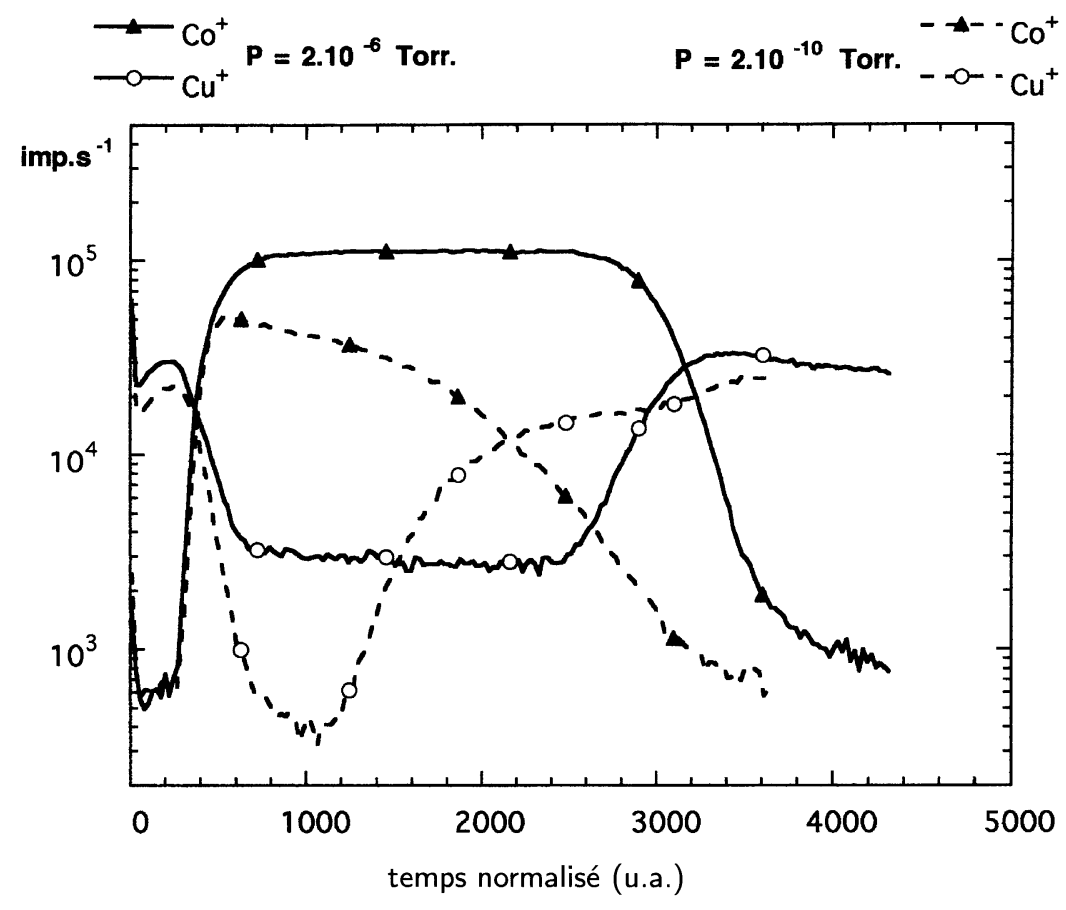

Fig. 8. - Influence de la pression de soufflage à l'oxygène sur deux profils obtenus sur un même échantillon.

[Comparison of two profiles obtained under different flooding pressures.]

Tableau II

\begin{tabular}{|c|c|c|c|c|}
\cline { 3 - 5 } \multicolumn{2}{c|}{} & $Z_{\max }$ & $R_{\mathrm{a}}(\mathrm{nm})$ & $R_{\mathrm{q}}(\mathrm{nm})$ \\
\hline Surface de départ & & 13 & 1,6 & 2,1 \\
\hline incidence tangentielle & $\mathrm{Cu}$ & 130 & 20 & 23 \\
\cline { 2 - 5 } mauvaise résolution & $\mathrm{Co}$ & 61 & 4 & 5 \\
\hline $\begin{array}{c}\text { incidence normale } \\
\text { meilleure résolution }\end{array}$ & $\mathrm{Cu}$ & 81 & 6 & 8 \\
\cline { 2 - 5 } & $\mathrm{Co}$ & 30 & 3,8 & 5 \\
\hline
\end{tabular}

Des analyses statistiques des images topographiques, nous retiendrons pour comparer les fonds de cratères les trois paramètres suivants :

- les dénivelés maximums dans l'image : $z_{\max }$;

- la rugosité moyenne - valeur moyenne des hauteurs par rapport au plan moyen : $R_{\mathrm{a}}$;

- la déviation standard des hauteurs par rapport au plan moyen : $R_{\mathrm{ms}}-R_{\mathrm{q}}$.

Les résultats sont reportés sur le tableau II.

À l'examen de ce tableau il apparaît que la pulvérisation entraîne l'apparition d'une rugosité induite. Cette rugosité est beaucoup plus importante lorsque la pulvérisation est effectuée sous une incidence tangentielle, ce qui est cohérent avec l'évolution des observations microscopiques MEB. 


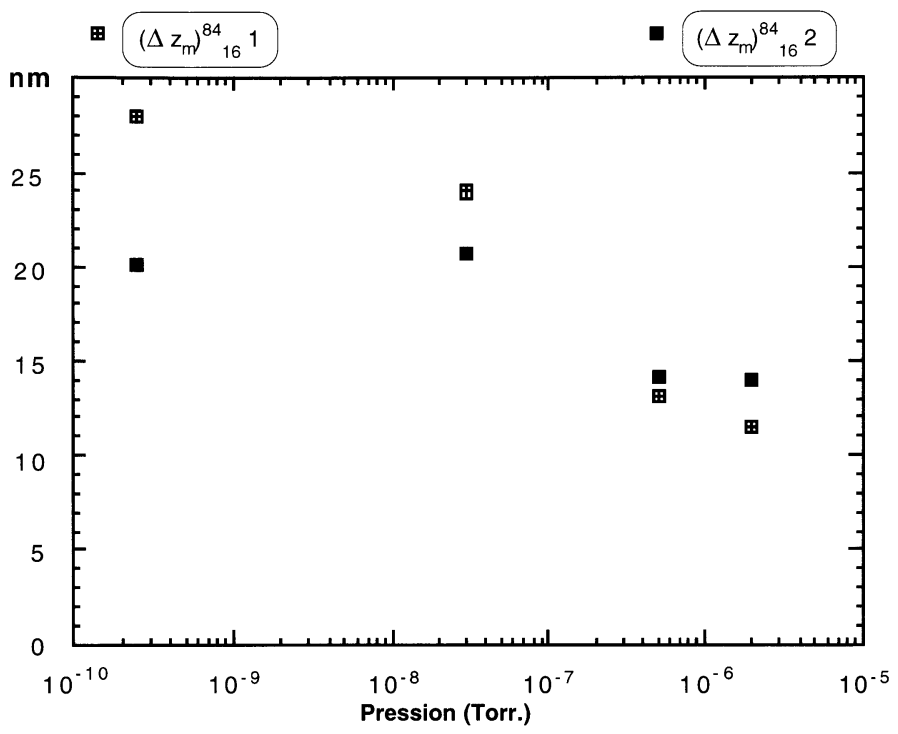

Fig. 9. - Résolutions moyennes $\left(\Delta z_{\text {moyen }}\right)_{16}^{84}$ pour les deux interfaces en fonction de la pression de soufflage à l'oxygène. Déterminations effectuées à $\pm 0,5 \mathrm{~nm}$.

[Mean depth resolution $\Delta z_{\text {mean }}$ for the two interfaces as a function of flooding pressure. Experimental error $\pm 0.5 \mathrm{~nm}$.]

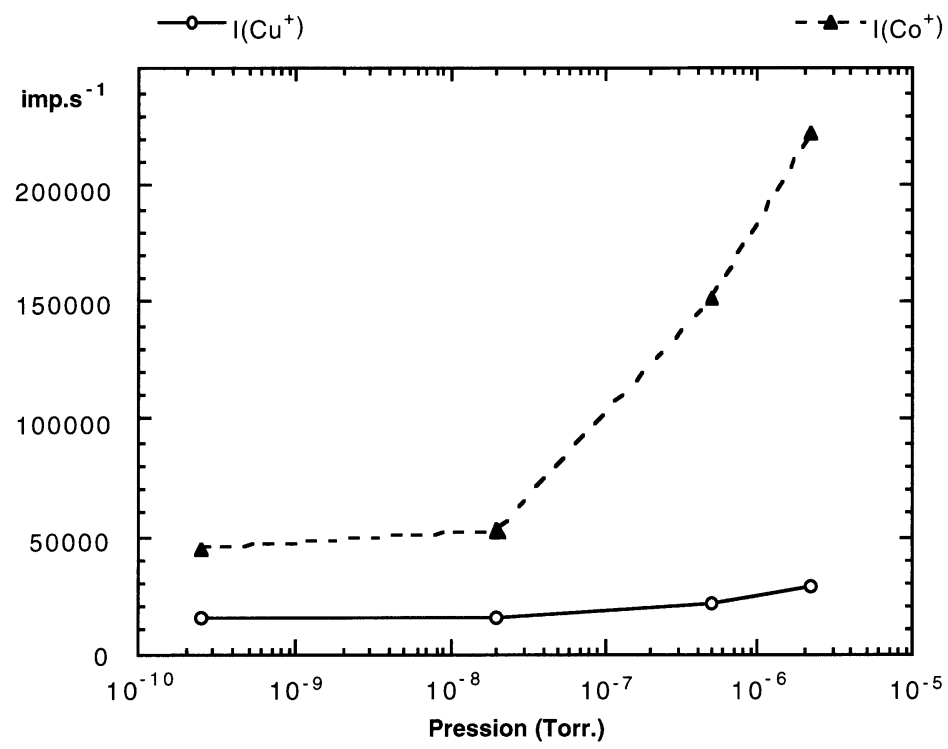

Fig. 10. - Évolution des intensités ioniques secondaires $\mathrm{Cu}^{+}$et $\mathrm{Co}^{+}$en fonction de la pression de soufflage à l'oxygène.

[Evolution of the $\mathrm{Cu}^{+}$and $\mathrm{Co}^{+}$secondary ions intensities versus flooding pressure.] 


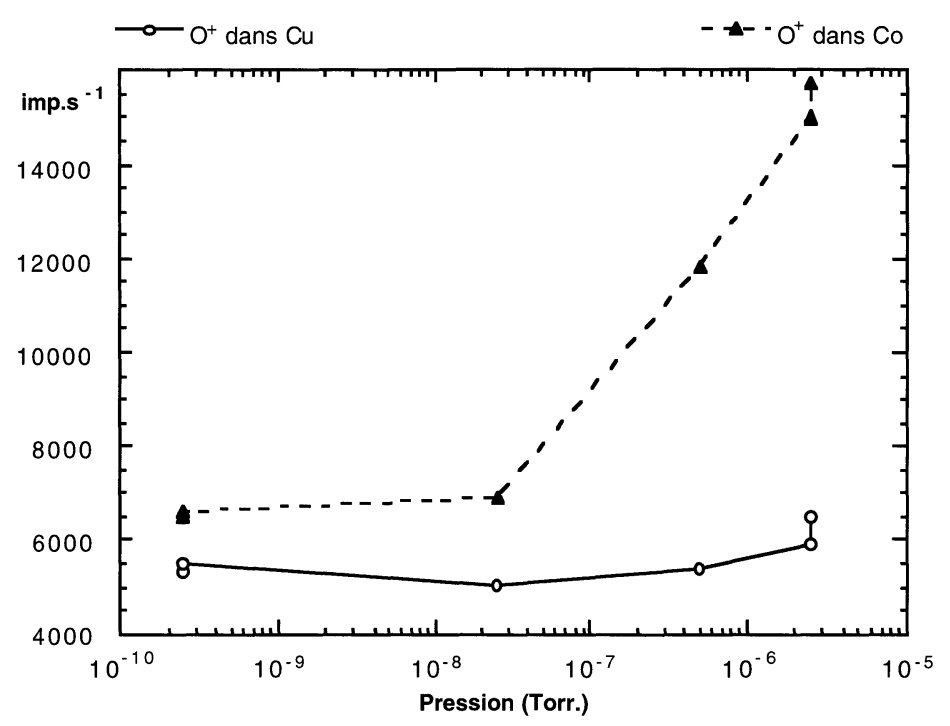

Fig. 11. - Évolution de l'intensité ionique secondaire $\mathrm{O}^{+}$mesurée dans le cuivre et le cobalt en fonction de la pression de soufflage à l'oxygène.

[Evolution of the $\mathrm{O}^{+}$secondary ion intensity measured in copper and cobalt versus flooding pressure.]

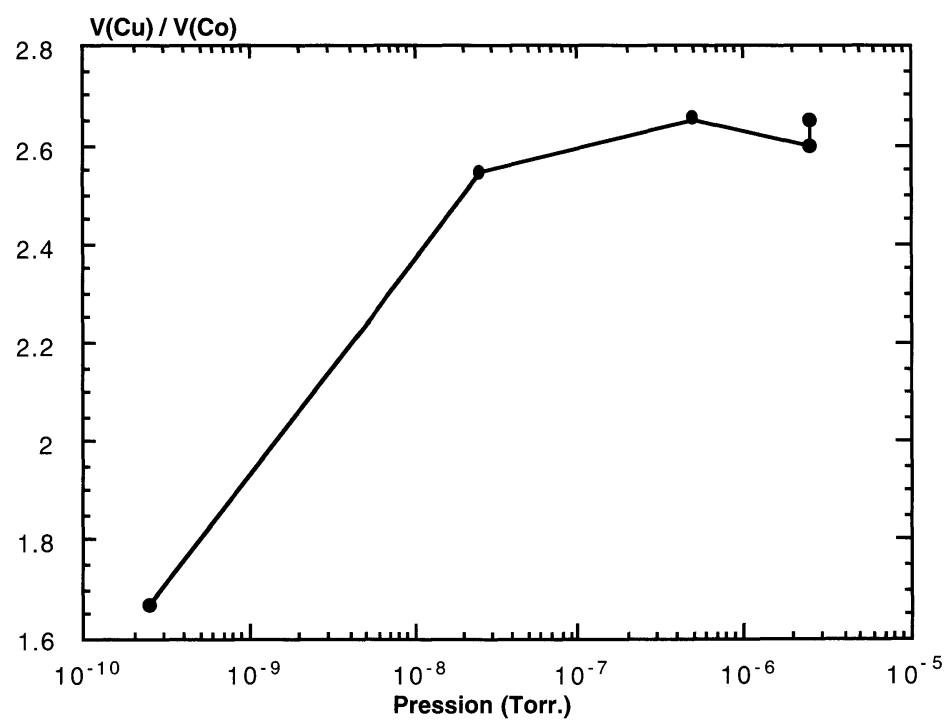

Fig. 12. - Évolution du rapport de la vitesse de pulvérisation du cuivre sur celle du cobalt en fonction de la pression de soufflage à l'oxygène.

[Variation of sputtering rates ratio of copper/cobalt as a function of the flooding pressure.] 


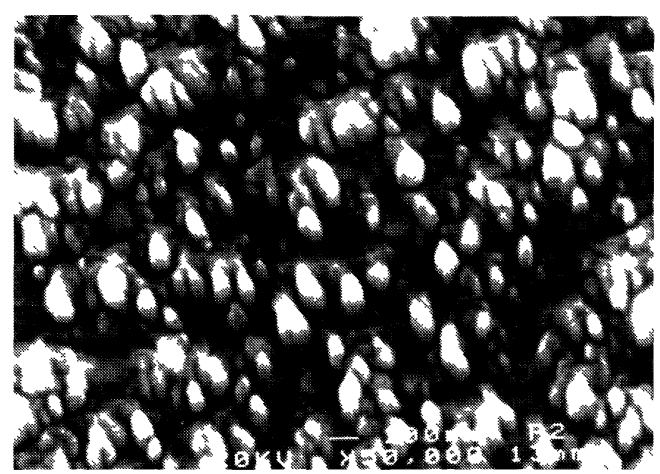

a)

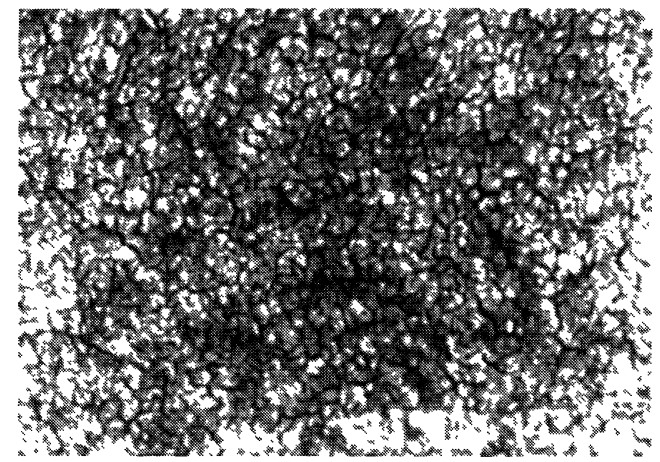

b)

Fig. 13. - Examen par microscopie électronique à balayage des fonds de cratères de pulvérisation: a) cuivre, b) cobalt.

[SEM micrograhies of cratere bottom for two kind of sample: a) copper, b) cobalt.]

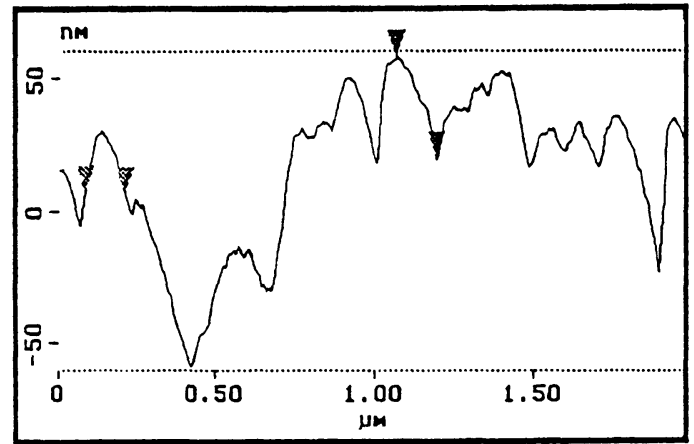

a)

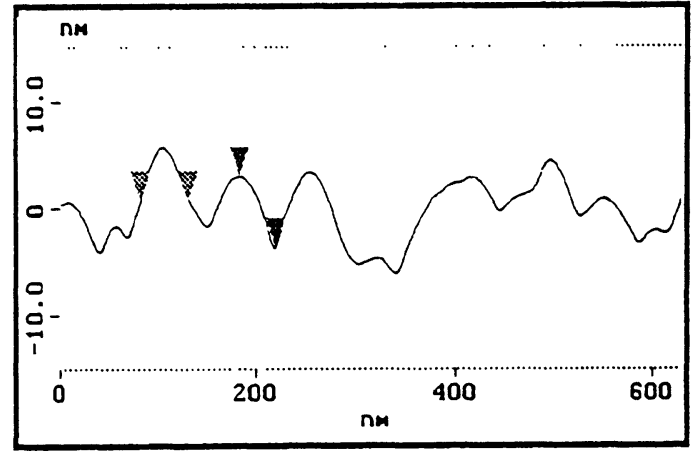

b)

Fig. 14. - Profils de rugosité enregistrés par AFM sur des fonds de cratères de natures différentes: a) cuivre, b) cobalt. Ces profils correspondent aux photographies MEB (noter le changement de l'échelle en $z$ ).

[Roughness profils measured by AFM on cratere bottom of different composition: a) copper, b) cobalt. These profils are related with the SEM micrographies (notice the modification of the $z$ scale).]

Fait important à noter, le cuivre, avec des conditions de pulvérisation identiques, présente toujours une rugosité supérieure à celle du cobalt. Sur les mêmes échantillons cette observation à également été signalée par Grattepain et al. [5].

\section{4. Évolution des signaux SIMS aux interfaces}

Les deux éléments (Co et $\mathrm{Cu}$ ) constitutifs des interfaces sont assez voisins dans la classification périodique. Néanmoins les métaux présentent des différences de propriétés pouvant intervenir lors de la pulvérisation d'une interface. On peut retenir d'une part des vitesses de pulvérisation différentes $\left(V_{\mathrm{p}}(\mathrm{Cu})>V_{\mathrm{p}}(\mathrm{Co})\right)$, d'autre part des solubilités mutuelles différentes également. En effet, le cuivre est plus soluble dans le cobalt $(2 \%)$ que le cobalt dans le cuivre $(0,1 \%)$. Ces différences entre les deux métaux pourraient entraîner des "dissymétries" entre les réponses de la SIMS lors du passage des interfaces $\mathrm{Co} \rightarrow \mathrm{Cu}$ et $\mathrm{Cu} \rightarrow \mathrm{Co}$. 


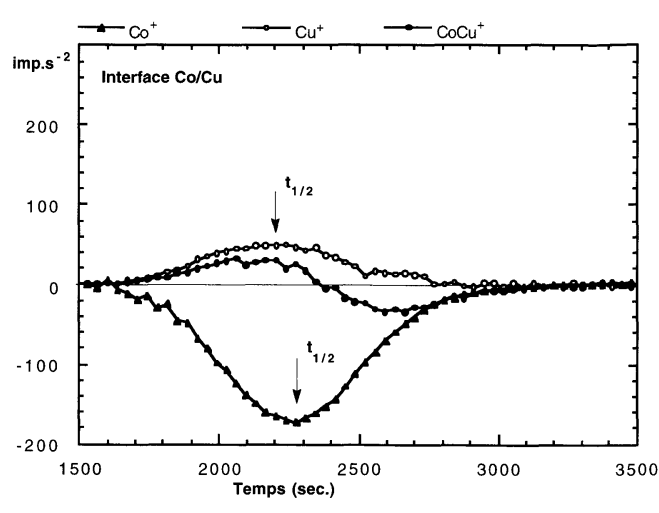

a)

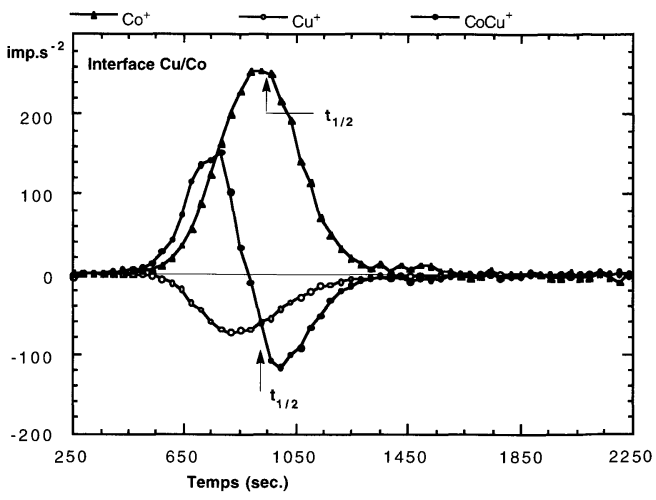

b)

Fig. 15. - Dérivées des signaux $\mathrm{Cu}^{+}, \mathrm{Co}^{+}, \mathrm{CoCu}^{+}$, en fonction du temps lors du passage d'une interface $\mathrm{Co} / \mathrm{Cu}$ et d'une interface $\mathrm{Cu} / \mathrm{Co}$. Les valeurs des $t 1 / 2$, mesurés de façon conventionnelle sont égalemment reportés sur les graphes.

[Derivatives of the $\mathrm{Cu}^{+}, \mathrm{Co}^{+}, \mathrm{CoCu}^{+}$intensities versus time for $\mathrm{Co} / \mathrm{Cu}$ and $\mathrm{Cu} / \mathrm{Co}$ interfaces. The $t 1 / 2$ values are also reported.]

Nous avons comparé deux premières interfaces $(z=30 \mathrm{~nm})$, une interface $\mathrm{Co} \rightarrow \mathrm{Cu}$ et une interface $\mathrm{Cu} \rightarrow$ Co. Les évolutions en fonction du temps des dérivées des signaux $\mathrm{Co}^{+}, \mathrm{Cu}^{+}$ et $\mathrm{CoCu}^{+}$sont présentées figure 15 , pour des conditions expérimentales identiques $\left(\mathrm{O}_{2}^{+}\right.$, angle d'incidence $8^{\circ}$, soufflage à l'oxygène). Bien que ces courbes se rapportent à des interfaces de natures différentes $(\mathrm{Co} \rightarrow \mathrm{Cu}$ et $\mathrm{Cu} \rightarrow \mathrm{Co}$ ), celles-ci sont très semblables. on peut noter que les maximums des dérivées des ions secondaires $\mathrm{Co}^{+}$et $\mathrm{Cu}^{+}$ne coïncident pas exactement dans le temps. Le maximum de la dérivée du signal $\mathrm{Cu}^{+}$précède légèrement et de façon systématique, le maximum de la dérivée du signal $\mathrm{du} \mathrm{Co}^{+}$. Ces courbes représentent des variations en fonction du temps. En toute rigueur la transformation de la coordonnée temps en une coordonnée de profondeur fait intervenir une vitesse de pulvérisation variable lors du passage de l'interface : celle-ci augmente pour l'interface $\mathrm{Co} \rightarrow \mathrm{Cu}$ et diminue pour l'interface $\mathrm{Cu} \rightarrow \mathrm{Co}$. Cette transformation aurait pour effet de dissymétriser les courbes obtenues mais ne changerait pas l'ordre de la succession en fonction du temps des maximums des dérivées. Un effet de matrice sur les coefficients d'ionisation secondaire des atomes métalliques peut expliquer ce décalage temporel entre les maximums des dérivées. Le cuivre est plus électronégatif que le cobalt $(E(\mathrm{Cu})=1,9, E(\mathrm{Co})=1,8)$ ce qui pourrait induire une atténuation du signal du cuivre relativement à celui du cobalt dans la zone de l'interface. La vérification de cet effet de matrice nécessite un support expérimental qui sort du cadre de cette étude.

\section{Conclusions}

Dans cette étude nous avons effectué une étude méthodologique de la profilométrie par SIMS sur des échantillons présentant des couches alternées de cuivre et de cobalt avec une épaisseur totale de $100 \mathrm{~nm}$. Compte tenu de l'absence d'autre caractérisation à l'échelle atomique des interfaces étudiées nous n'avons pu que "qualifier" les profils et en déduire l'influence des conditions expérimentales de pulvérisation sur la résolution en profondeur obtenue. 
Les profils ont été réalisés en pulvérisant avec des ions primaires réactifs $\mathrm{O}_{2}^{+}$et d'énergie $3 \mathrm{keV}$. Les résultats obtenus montrent clairement que la meilleure résolution en profondeur est obtenue sous incidence normale. Sous incidence tangentielle la résolution se dégrade très rapidement en fonction de la profondeur érodée. Avec nos échantillons, à une profondeur de 80 $\mathrm{nm}$, la deuxième interface ne se matérialise plus sur les profils. Le soufflage à l'oxygène permet dans une large mesure d'éviter cette dégradation de la résolution.

La résolution en profondeur est conditionnée par la rugosité induite lors de la pulvérisation. Cette rugosité induite est en partie éliminée par implantation d'oxygène. L'implantation d'oxygène due aux seuls ions $\mathrm{O}_{2}^{+}$de pulvérisation est suffisante sous incidence normale. Sous incidence tangentielle il est nécessaire de souffler à l'oxygène pour l'augmenter .

En ce qui concerne les vitesses de pulvérisation, leur évolution montre que les variations de $S(\mathrm{Co})$ et $S(\mathrm{Cu})$ en fonction de l'angle ne suivent pas une loi en $\cos (\theta)^{-n}$ (avec $n \sim 1$ ). D'autre part le rapport $S(\mathrm{Cu}) / S(\mathrm{Co})$ est fonction de l'angle d'incidence des ions de pulvérisation ce qui indique des variations de $S$ en fonction de $\delta$ différentes pour les deux métaux [6].

Les topographies du cuivre et du cobalt, après des pulvérisations équivalentes, sont très différentes. Sur les échantillons que nous avons utilisés, obtenus par pulvérisation cathodique $\mathrm{RF}$, le cuivre présente systématiquement une rugosité plus développée que le cobalt. Ce dernier point mériterait une confirmation sur des matériaux massifs et purs.

\section{Remerciements}

Les auteurs remercient,

- H. Le Gall - Laboratoire de physique des solides de Bellevue - CNRS 1 place A. Briand, 92195 Meudon Élaboration des échantillons.

- T. David - Équipe Submicronique. LPUB - Université de Bourgogne Mesures de Rugosité - Microscopie AFM.

- S. Collin - UMR 5613 CNRS - Université de Bourgogne Photographies MEB.

- Tous leurs collègues du GDR (G1108) : Caractérisation des interfaces dans les multimatériaux.

\section{Bibliographie}

[1] Wittmaack K., Practical surface analysis, Briggs and Seah Eds. (Wiley J., 1992) Tome 2, Chap 3.

[2] Hofmann S., Appl. S.S. 70/71 (1993) 9-19.

[3] Dupuy J.C., Dubois C., Prudon G., Brenier R. et Thevenard P., Nucl. Instr. Method. B 64 (1992) 636-640.

[4] Grattepain C., Dubois C., Tromson-Carli A. et Ballutaud D., 1er Colloque de la Société Française des Microscopies (SF $\mu$ ), Rennes, Juin 1996.

[5] Grattepain C., Dubois C. et Repoux M., Colloque Interfaces et Multimatériaux (I2M), Aix-en-Provence, Mai 1997.

[6] Drisse O. et Blaise G., Colloque Interfaces et Multimatériaux (I2M), Aix-en-Provence, Mai 1997. 